Vol. 19, n² | 2015

Varia

\title{
Jason Pine, The Art of Making Do in Naples
}

Minneapolis-London, University of Minnesota Press, 2012, 360 p., ISBN

0816676011

\section{Clotilde Champeyrache}

\section{(2) OpenEdition}

\section{Journals}

Édition électronique

URL : http://journals.openedition.org/chs/1614

DOI : $10.4000 /$ chs. 1614

ISSN : 1663-4837

Éditeur

Librairie Droz

\section{Édition imprimée}

Date de publication : 1 novembre 2015

Pagination : 156-158

ISBN : 978-2-600-01908-8

ISSN : $1422-0857$

Référence électronique

Clotilde Champeyrache, "Jason Pine, The Art of Making Do in Naples », Crime, Histoire \& Sociétés / Crime, History \& Societies [En ligne], Vol. 19, n² | 2015, mis en ligne le 01 novembre 2017, consulté le 24 septembre 2020. URL : http://journals.openedition.org/chs/1614 ; DOI : https://doi.org/10.4000/ chs. 1614

Ce document a été généré automatiquement le 24 septembre 2020.

(c) Droz 


\title{
Jason Pine, The Art of Making Do in Naples
}

\author{
Minneapolis-London, University of Minnesota Press, 2012, 360 p., ISBN
}

0816676011

\section{Clotilde Champeyrache}

\section{RÉFÉRENCE}

Jason Pine, The Art of Making Do in Naples, Minneapolis-London, University of Minnesota Press, 2012, 360 p., ISBN 0816676011.

1 Depuis les années quatre-vingt-dix, un style musical domine dans les rues de Naples : la chanson néo-mélodique (canzone neomelodica). Populaire, strictement en dialecte napolitain, mélange de rythmes pop et de sonorités orientalisantes, la chanson néomélodique suscite les passions en Campanie et, pour d'autres raisons, dans le reste de l'Italie. En Campanie, elle constitue une affirmation culturelle et identitaire, un espoir de connaître peut-être un jour la gloire ou au moins une façon de s'en sortir dans la vie, mais aussi toute une industrie largement souterraine. Dans le reste de l'Italie, à l'exception de zones comme la Sicile ou le sud de Rome, elle est souvent la risée des critiques musicaux ou dénoncée comme singulièrement proche des valeurs criminelles véhiculées par la camorra, la mafia napolitaine. Rares sont les chanteurs néomélodiques à connaître - comme Nino d'Angelo ou Gigi d'Alessio - une gloire nationale. Pourtant, ils sont nombreux dans la cité parthénopéenne ceux qui se lancent dans cette voie et qui vivent - plus ou moins bien - de leurs performances artistiques entre ventes de $\mathrm{CD}$, passages à répétition sur des télés pirates et prestations à la chaîne à l'occasion de mariages, baptêmes et autres cérémonies. C'est que, derrière ces chanteurs, prospère une véritable industrie musicale aux revenus non négligeables et qui met en jeu des studios d'enregistrement plus ou moins légaux, des chaînes de télévision non autorisées basées dans des appartements, des salles de spectacle et boîtes de nuit en location, des tournages de clips pour inonder youtube et facebook. 
2 C'est ce monde des chanteurs néo-mélodiques que Jason Pine, anthropologue à l'université de New York, explore dans The Art of Making Do in Naples. Exploration au sens effectif du terme puisque l'auteur réalise un travail de terrain, d'immersion même, de plusieurs années dans les quartiers populaires de Naples. Parti pour apprendre le dialecte napolitain, il ne peut que découvrir sur place la musique néo-mélodique non pas tant comme spectateur que comme «art de la débrouille " pratiqué à large échelle et auquel Jason Pine lui-même va s'exercer. Rapidement, le séjour napolitain et la découverte de l'industrie néo-mélodique dévoilent une autre présence à la fois impalpable et inéluctable, celle de la camorra. C'est ce que l'auteur analyse en termes de «zone de contact», cette zone grise où "les économies formelle, informelle et souterraine se superposent » (p. 62). C'est également là que résident la force et l'intérêt principal de l'ouvrage. Loin des clichés sur la mafia tentaculaire, pyramidale et manichéenne, la camorra est présentée dans sa réalité territoriale et dans sa spécificité de mafia en tant que forme particulière et ultime de criminalité organisée.

3 Ce qui frappe en tout premier lieu, et qui est un trait saillant des criminalités mafieuses, c'est la quotidienneté de la camorra, c'est-à-dire son omniprésence réelle ou simplement suggérée dans le quotidien des habitants de Naples. La camorra n'est pas l'expression d'une déviance criminelle qui ne serait concrète que pour ceux qui choisissent de vivre en marge. Elle est, au contraire, parfaitement insérée dans la vie et l'économie locales. Même le citoyen honnête - par exemple celui qui essaie de s'en sortir avec les moyens du bord - peut être amené à côtoyer le camorriste, voire faire affaire avec lui, de plus ou moins bon gré. Le camorriste est, en effet, l'entrepreneur, celui qui peut faire démarrer votre carrière, vous ouvrir les portes des cérémonies de familles mafieuses, vous faire enregistrer de meilleurs clips que la concurrence, vous rémunérer aussi avec de la drogue ou vous racketter sur le cachet de vos prestations. À ce jeu-là la question du « qui es-tu et qui suis-je ?» (p. 249) devient fondamentale entre ceux qui se prétendent camorristes, ceux qui le sont vraiment et ceux désireux d'entrer sur la scène - très théâtrale - de la chanson néo-mélodique. Jason Pine, entré de plainpied dans cette industrie musicale en tant que réalisateur de clips, va devoir se confronter à cette question, réaliser quelques faux pas, maîtriser certains codes tacites et finir par s'approcher d'agents camorristes même si, au fond, comme dans toute zone grise, un doute subsiste toujours sur qui est réellement qui.

La camorra est également présentée de façon très juste comme une forme entrepreneuriale, une capacité permanente à saisir des opportunités économiques, à générer du profit, de façon légale éventuellement, de manière illégale si nécessaire. Le marché est loin d'être négligeable entre la vente de CD et les concerts privés ; certains chanteurs néo-mélodiques vendant plus à Naples que des chanteurs à l'audience nationale. Impossible donc, pour une organisation mafieuse, de passer à côté de ce secteur. D'autant plus, même si cela ne transparaît pas dans le récit de Jason Pine, qu'il s'agit d'une industrie clairement liée au territoire et donc à son contrôle à travers aussi bien les emplois créés que les fans drainés par les chanteurs en vogue.

Un autre point fort indéniable dans la restitution du phénomène camorriste est l'accent mis sur l'importance des réseaux au lieu de la fable sur une mafia napolitaine très structurée, hiérarchisée, centralisée. Cela permet de comprendre comment la camorra réussit à être présente dans la vie quotidienne de la population, comment légal et illégal s'enchevêtrent en permanence, pourquoi aussi le système est aussi souple et pérenne dans le temps. Face aux dénonciations, aux descentes de police, l'industrie 
néo-mélodique perdure et l'association criminelle également. Lorsqu'un appartement servant à l'enregistrement d'une télé pirate est perquisitionné par les forces de l'ordre, les dégâts sont limités car l'«art de la débrouille » a déjà permis la mise en place d'un nouveau studio d'enregistrement clandestin. De même, la mise en contact avec la camorra n'est pas frontale comme elle le serait si l'organisation était pyramidale et rigide. Tout se fait par une chaîne de contacts supposée aboutir à la rencontre avec un boss local. Et cette chaîne est en partie symbolique des différents degrés de proximité qu'un habitant des quartiers populaires de Naples peut avoir avec la mafia - du rejet jusqu'à la complicité - mais aussi du caractère parfois involontaire de la fréquentation mafieuse : le camorriste n'est pas un être à part auréolé de son pouvoir criminel, il peut être le voisin de palier à la mine commune.

6 Si l'ouvrage constitue une impressionnante plongée dans la réalité napolitaine et son inévitable camorra, il n'en reste pas moins sujet à quelques critiques. Ainsi certaines digressions sur la vie personnelle de l'auteur ne sont pas toujours, voire pas du tout essentielles à la compréhension de l'analyse de terrain et de ses fruits. Un cadrage plus strict aurait peut-être réduit le volume publié (303 pages sans les notes et avec des photos en noir et blanc souvent intéressantes) mais aurait probablement rendu le contenu plus dynamique. Cela pose d'ailleurs la question de la construction du texte, pas forcément la plus lisible pour un lecteur standard non spécialiste du sujet et/ou en anthropologie. Par moments, une sensation de flottement peut en effet saisir le lecteur, une impression de ne plus savoir où l'on en est dans le récit des aventures de terrain de l'auteur. Certes, on pourra arguer que cela reflète également les interrogations $d u$ chercheur face à une quête - celle du contact camorriste - dont l'objet ne cesse de se dérober ou d'apparaître mais sans dire son nom. Au fond, la structure déconcertante de l'ouvrage pourrait bien transcrire de façon scripturale l'impalpable omniprésence de la camorra.

7 Enfin, quitte à raisonner en termes de « zones de contact ", l'ouvrage aurait sans doute tiré bénéfice - éventuellement sous la forme d'un chapitre dédié - d'un approfondissement des textes des chansons néo-mélodiques, certaines constituant une véritable apologie de la camorra et une dénonciation des collaborateurs de justice (les mal-nommés «repentis») et des forces de l'ordre et de la justice. Dans ce cas, la chanson néo-mélodique prend une dimension supplémentaire, et inquiétante étant donné sa popularité, puisque d'industrie rentable elle devient outil de promotion d'un idéal mafieux de "criminalité honorable» et rejoint une tradition de "chants de mauvaise vie» au même titre que les narco-corridos, les «ballades de la drogue» mexicaines.

8 En somme, Jason Pine explore un monde fascinant et nous fait partager un travail de terrain d'une extrême densité et originalité. Et, finalement, même dans ses quelques défauts, il donne envie d'en savoir plus sur le sujet, d'aller écouter cette Naples souterraine qui échappe souvent aux touristes alors même que la chanson néomélodique constitue la bande son des rues parthénopéennes. Rien n'empêche alors le lecteur italophone de compléter cette lecture par celle de Marcello Ravveduto, Napoli... Serenata Calibro 9 (Liguori, Naples, 2007). 


\section{AUTEURS}

\section{CLOTILDE CHAMPEYRACHE}

Université Paris 8 